Article

\title{
Effect of a Synbiotic Mix on Lymphoid Organs of Broilers Infected with Salmonella typhimurium and Clostridium perfringens
}

\author{
Zuamí Villagrán-de la Mora ${ }^{1,2}{ }^{\oplus}$, Olga Vázquez-Paulino ${ }^{3}$, Hugo Avalos ${ }^{3,+}{ }^{\dagger}$, Felipe Ascencio ${ }^{2}$, \\ Karla Nuño ${ }^{4, *}$ and Angélica Villarruel-López ${ }^{4, *}$ \\ 1 Departamento de Ciencias de la Salud, Centro Universitario de Los Altos, Universidad de Guadalajara, \\ Av. Rafael Casillas Aceves 1200, Tepatitlán de Morelos 47620, Jalisco, Mexico; \\ blanca.villagran@academicos.udg.mx \\ 2 Centro de Investigaciones Biológicas del Noroeste (CIBNOR), Av. Instituto Politécnico Nacional 195, \\ Playa Palo de Santa Rita Sur, La Paz 23096, BCS, Mexico; ascencio@cibnor.mx \\ 3 Departamento de Farmacobiología, Centro Universitario de Ciencias Exactas e Ingenierías, Universidad de \\ Guadalajara, Blvd. Gral. Marcelino García Barragán 1421, Olímpica, Guadalajara 44430, Jalisco, Mexico; \\ olga.vazquez@academicos.udg.mx (O.V.-P.); avalossanchez@gmail.com (H.A.) \\ 4 Departamento de Ciencias Biomédicas, Centro Universitario de Tonalá, Universidad de Guadalajara, \\ Nuevo Perif. Ote. 555, Ejido San José Tateposco, Tonalá 45425, Jalisco, Mexico \\ * Correspondence: karlajanette.nuno@cutonala.udg.mx (K.N.); angelica.vlopez@academicos.udg.mx (A.V.-L.) \\ + This study is dedicated in memory of the late Dr. Hugo Avalos.
}

Received: 26 February 2020; Accepted: 16 May 2020; Published: 19 May 2020

Simple Summary: The use of synbiotics in the poultry industry could be a tool to regulate immunological activity and generate beneficial effects against pathogens, like Salmonella typhimurium and Clostridium perfringens, particularly in those cases where the use of antibiotics during poultry production was excluded. Either through the generation of short-chain fatty acids (SCFA) that contribute to mucosa proliferation or promoting the growth of beneficial gut bacteria, synbiotics could favor a microenvironment that improves the activity of the immune system. However, the organization and response of lymphocytes in lymphoid tissues could be modified by the type of active compound of the synbiotic. Therefore, the present work investigated the effect of a synbiotic mix on lymphoid tissues of broilers infected with Salmonella typhimurium and Clostridium perfringens. The results showed that the mix of probiotics Lactobacillus rhamnosus HN001, Pediococcus acidilactici MA18/5Ma and a prebiotic can stimulate the bursa and the IgA production, increasing the size of its follicles and promoting the ability to resist infections caused by $S$. typhimurium in broilers.

Abstract: Synbiotic consumption can modulate immune response. This work involves studying the effect of a synbiotic on lymphoid organs and IgA of broilers infected with Salmonella typhimurium and Clostridium perfringens. A total of 258 one-day-old male broilers (Gallus gallus domesticus), line COBBAvian48 (free of growth-promoting antibiotics), were distributed into eight treatment groups. A symbiotic mix comprising Lactobacillus rhamnosus HN001 and Pediococcus acidilactici MA18/5 M as probiotics and $4.5 \%\left(0.045 \mathrm{~g} \mathrm{~g}^{-1}\right)$ of Agave tequilana fructans as prebiotic per dose (one milliliter) was administered through drinking water the first day of life. Bursa, spleen and thymus were analyzed. Broilers treated with the synbiotic, whether or not infected with pathogens, had bigger bursa follicles than the non-treated $(p<0.05)$, and the ones from the synbiotic group had more lymphocytes than the control group $(p<0.05)$. Thymus follicles of the synbiotic group were bigger than the control group $(p<0.05)$. Lesions associated with Salmonella infection were found in the bursa, however, in the broilers treated with the synbiotic, the lesions were less intense and were not present after 32 days of life. The synbiotic mix can stimulate the bursa, increasing the size of their follicles and promoting the ability to resist infections caused by $S$. typhimurium in broilers. 
Keywords: probiotic; prebiotic; Clostridium perfringens; Salmonella typhimurium; bursa; spleen; thymus

\section{Introduction}

The poultry industry has been using synthetic antibiotics for immune modulation as feed additives to control pathogens growth [1]. However, because of their effect on promoting antibiotic resistance of microorganisms present in animals [2,3], antibiotics in feces and urine that can spread in the environment [2,4] and in poultry meat [3,5]; and the consumer demand for access to "natural" and antibiotic-free products [6], there is a trend towards decreasing the use of antibiotics as growth promoters [7].

Salmonella is a Gram-negative rod-shaped bacteria [8]. The genus Salmonella comprises three species, Salmonella enterica, Salmonella bongori and Salmonella subterranean. S. enterica, is further classified into six subspecies: enterica (subsp. I), arizonae (subsp. IIIa), diarizonae (subsp. IIIb), houtenae (subsp. IV), indica (subsp. VI) and salamae (subsp. II); with more than 50 serogroups and over 2500 serotypes [9]. Salmonella enterica subsp. enterica serovar typhimurium is the causal agent of white diarrhea [7].

Clostridium perfringens is a Gram-positive anaerobic spore-forming bacterium, able to produce various toxins and enzymes responsible for the associated lesions and symptoms. $C$. perfringens strains are classified into five toxinotypes (A, B, C, D and E), based on the production of four major toxins $(\alpha, \beta$, $\epsilon$ and ı). C. perfringens type A causes necrotic enteritis and the subclinical form infection in poultry [4].

Excluding antibiotics as growth promoters during poultry production increases the incidence of certain animal diseases such as those caused by S. typhimurium and C. perfringens [7]. It has been suggested that synbiotics could offer resistance to gut bacterial infections working as an antibacterial and under normal conditions, probiotics and prebiotics can improve growth performance, intestinal microbial ecology and immunity of poultry $[10,11]$.

The immune system of poultry is a complex, multi-factorial entity. In chickens, comprises primary (bursa of Fabricius and thymus) and secondary (spleen, Harderian gland and mucosa-associated lymphoid tissue [MALT]) immune organs [12]. The immune function is affected by bird age, diet composition, feed and energy intake, genetic potential for growth, environment and stress, among others. This plasticity and responsiveness to external influences have led to many efforts over the years to manipulate immune function through vaccination, reduction or elimination of specific pathogens (e.g., biosecurity), dietary growth promoting antibiotics, nutritional immunomodulation and administration of synbiotics [13].

The intestine — and the microbiota that lives in it—plays an important role in shaping the innate and adaptive immune system $[14,15]$. It was shown that oral administration of probiotics can significantly affect the systemic and mucosa-associated immune responses, resulting in disease prevention [6]. Probiotics interact with monocytes/macrophages, lymphocytes, dendritic cells and intestinal epithelial cells. Dendritic cells and intestinal epithelial cells can interact and respond to intestinal pathogens through their pattern recognition receptors [16]. Studies with L. rhamnosus have shown that this microorganism enhances the expression of TLR9, which was identified as a tumor necrosis factor (TNF) attenuator, showing an inhibitory effect on the reduction of TNF- $\alpha$ induced transepithelial resistance [17].

Poultry production is considered a "stressful system" that acts on birds by generating stress responses with neuroendocrine and metabolic changes [18]. During these responses, some physiological activities like growth, reproduction and digestion may be totally or partially withdraw, directing energy resources to meet other organs' demands, such as the central nervous system and skeletal muscle. Usually, adrenal hypertrophy coexists with involution of the lymphoid organs, including atrophy of the thymus, bursa, spleen and pancreas, usually larger and faster growing birds are more sensitive to that condition [19]. 
Studies have tried to modify the immune function of chickens through nutritional compounds like fatty acids and vitamin E, prebiotics, probiotics and synbiotics. However, so far, the effect of synbiotics on the immune system has not been clarified, because while there are studies that indicate that it is possible to modify the immune response through food additives, others report no change at the time of administration [13]. Therefore, the present study was conducted to evaluate the effect of a synbiotic formulated with agave inulin as a prebiotic and Lactobacillus rhamnosus and Pediococcus acidilactici as probiotics on lymphoid organs and IgA of broilers infected with Salmonella typhimurium and Clostridium perfringens.

\section{Materials and Methods}

\subsection{Experimental Birds and Housing}

The present study was carried out following the guidelines of the Institutional Animal Care and Use Committee (IACUC) and was approved by the Bioethics Committee (CUCBA) of the University of Guadalajara (Permit Number: CINV.078/15).

Two hundred and fifty-eight one-day-old male broilers (Gallus gallus domesticus), line COBBAvian48 (free of growth-promoting antibiotics), obtained from a local commercial hatchery (AVI-INC, Jalisco, Mexico) were housed and distributed randomly into eight treatment groups with three replicates each one. Each replicate was assigned to a pen physically separated from the other two of the same replicates and randomly placed in different sections of the shed. The treatments were: (1) control group $(n=43)$; (2) synbiotic $(n=35) ;(3)$ synbiotic mix + S. typhimurium $(n=25) ;(4)$ synbiotic mix + C. perfringens $(n=25)$; (5) synbiotic mix + S. typhimurium $+C$. perfringens $(n=25)$; $(6)$ S. typhimurium $(n=30)$; $(7)$ C. perfringens $(n=30)$; and (8) S. typhimurium $+C$. perfringens $(n=45)$. The experiment was conducted using a randomized complete block design and the blocking variables were the experimental unit (pen of broilers) and the sampling time.

Housing and feeding were performed as Villagran-de la Mora et al [20]. Broilers were vaccinated against avian pox, Gumboro and Newcastle diseases, but they were not vaccinated against Salmonella or Clostridium.

\subsection{Synbiotic Mix and Pathogens Administration}

A synbiotic mix comprising $7 \log$ of CFU g ${ }^{-1}$ Lactobacillus rhamnosus HN001 and Pediococcus acidilactici MA18/5 M as probiotics and 4.5\% $\left(0.045 \mathrm{~g} \mathrm{~g}^{-1}\right)$ of Agave tequilana fructans as prebiotic per dose $(1 \mathrm{~mL})$ was administered in drinking water the first day of the broilers life. The water containers with the mix were available for $2 \mathrm{~h}$.

For treatment groups 3 to 8 , pathogens were administered on day 17 [21]. S. typhimurium was subcultured in lactose broth with yeast extract and C. perfringens ATCC 13,124 was subcultured in thioglycolate broth and incubated under anaerobic environment; pathogens were separated by centrifugation (thrice at $4000 \mathrm{~g}$ for $20 \mathrm{~min})$ and washed in physiological saline solution $(0.8 \% \mathrm{NaCl})$. The pellets were suspended in physiological saline solution and the number of bacteria in the suspension was calculated using a nephelometer (DensiCHEK, Model: OA009372, bioMérieux, Inc, Missouri, MO, USA). Finally, $5 \log$ CFU of S. typhimurium and/or $3 \log$ CFU of C. perfringens per bird were administrated through drinking water [22].

To calculate the synbiotic and pathogen intake, we considered that, broilers consume $1.12 \mathrm{~mL}$ and $25 \mathrm{~mL}$ of water per hour at 1 and 17 days old, respectively [23].

\subsection{Slaughter and Collection of Samples}

Body weight (BW) of each bird was measured weekly. Four sampling times were scheduled with three replicas from each treatment at 22, 32, 36 and 39 days of life. Broilers were euthanized by intraperitoneal injection of $3 \mathrm{~mL}$ per $2.5 \mathrm{~kg}$ of broiler, $6.3 \%$ sodium pentobarbital (PISA Agropecuaria, Guadalajara, Mexico). Cardiac puncture was performed to obtain blood samples [24], which were 
deposited in collection tubes with coagulation activator applied by spraying (red cap-BD Vacutainer) and centrifuged at $1600 \times g$ for $10 \mathrm{~min}$ to separate the serum, which was aliquoted and stored at $-20^{\circ} \mathrm{C}$ for later analysis [25]. Subsequently, broilers were eviscerated, and their bursa, spleen and thymus were removed.

\subsection{Lymphoid Organs Evaluation}

Bursa and spleen were weighed, and their morphometric index was calculated according to the following formula [26]:

$$
\text { Morphometric index }=\frac{\text { organ weight }(\mathrm{g})}{\text { body weight }(\mathrm{g})} \times 1000
$$

Samples of the organs were placed in formaldehyde at 10\% (Cat. No. 256462.0905, PanReacAppliChem, Darmstadt, Germany), dehydrated in alcohol (Cat. No. 89370-084, VWR, Radnor, PA, USA) cleared in xylene (Cat. No. 89370-088, VWR, PA) and imbedded in paraffin (Cat. No. P3683, Merck, Darmstadt, Germany). Tissue blocks were cut in a microtome (Cat. No. 4062, SLEE medical GmbH, Mainz, Germany) into 5-mm thick sections and stained with hematoxylin-eosin. Images were analyzed using the software Motic Images Plus 2.0 (Motic, Kowloon, Hong Kong).

Nine complete bursal and thymus follicles, corresponding to three birds per treatment per time, were observed with $4 \times$ panoramic objective (Optical microscope model E200 LED, Nikon, Shinagawa, Tokyo, Japan). The follicle section was selected for analysis when the cut passed through the central area of the follicle. Medullar follicular area was determined by a line crossing the basal membrane that divided the two follicular areas. Follicular cortex was determined as total follicular area minus medullar area in each follicle [27]. Lymphocyte counts were performed using the $100 \times$ panoramic. Four fields per follicle, two in the cortex and two in the marrow, were counted for the bursa and thymus.

Spleen samples were observed with 100× panoramic (Optical microscope model E200 LED, Nikon) and mature lymphocytes present in the white pulp were counted, reporting all found by field (nine fields per sample). The field was chosen in the area adjacent to some small-caliber blood vessel within the white pulp.

A qualitative analysis was also carried out in the three organs using the objectives $4 \times, 10 \times$ and $40 \times$.

\subsection{Quantification of $I g A$}

IgA was quantified in serum samples using the ELISA technique (enzyme-linked immunosorbent assay), in duplicates and following the protocol of the Chicken ELISA kit of Abcam IgA (Cat. No. 157691, Abcam, Cambridge, UK); the samples were diluted in $1 \times$ diluent (Abcam) and placed in the wells of the plate, incubated in darkness at room temperature for $20 \mathrm{~min}$. Once the incubation time was over, the content of the wells was aspirated, and three rinses were performed with the $1 \times$ Wash Buffer (Abcam). After removing the buffer, the enzyme-antibody conjugate $1 \times($ Abcam) was added to each well and incubated in darkness at room temperature for $20 \mathrm{~min}$. At the end of the incubation time, three washes were performed with the $1 \times$ Wash Buffer (Abcam) and the TMB substrate solution (Abcam) was added to each well, allowing it to incubate in darkness for $10 \mathrm{~min}$. At the end of the incubation time, the stop solution (Abcam) was added and the absorbance was quantified in a microplate reader at $450 \mathrm{~nm}$ (Model 680, Bio-Rad, Hercules, CA, USA).

\section{Results}

The consumption effect of a synbiotic mix on the morphology of the bursa of broilers was evaluated. At 22 days of life, the average morphometric index of the control group birds was $2.5 \pm 1.2$, however, it was reduced at 39 days, with an average of $1.1 \pm 0.3 \mathrm{~g}$. In the synbiotic group, the initial morphometric index was $1.5 \pm 0.6 \mathrm{~g}$ (22 days of life), increasing until the end of the bioassay ( 39 days of life) to reach an index of $2.3 \pm 0.6 \mathrm{~g}$. It must be noted that although no significant difference was found $(p>0.05)$, this 
value was higher than the other treatments at the end of the experiment (control $1.1 \pm 0.3$ g; synbiotic + S. typhimurium $0.5 \pm 0.3 \mathrm{~g} ; \mathrm{S}$. typhimurium $1.5 \pm 1.1 \mathrm{~g}$; synbiotic + C. perfringens $1.7 \pm 0.3 \mathrm{~g}$; C. perfringens $1.1 \pm 1.2 \mathrm{~g}$; synbiotic $+S$. typhimurium + C. perfringens $1.1 \pm 0.5 \mathrm{~g} ;$ S. typhimurium + C. perfringens $1.9 \pm 0.6)$ (Table 1).

Table 1. Bursa morphometric index of broilers treated with the synbiotic mix and inoculated with Salmonella typhimurium and Clostridium perfringens.

\begin{tabular}{ccccccccc}
\hline \multirow{2}{*}{ Days of Life } & \multicolumn{7}{c}{ Treatments } \\
\cline { 2 - 8 } & CT & SB & SBST & ST & SBCP & CP & SBSTCP & STCP \\
\hline 22 & $2.5 \pm 1.2$ & $1.5 \pm 0.6$ & $2.8 \pm 0.6$ & $1.6 \pm 0.7$ & $1.9 \pm 0.5$ & $2.6 \pm 0.2$ & $1.6 \pm 0.6$ & $1.3 \pm 0.1$ \\
32 & $2.3 \pm 0.2$ & $2 \pm 0.3$ & $1.9 \pm 0.5$ & $4.1 \pm 3.6$ & $2 \pm 1$ & $4 \pm 2$ & $2.8 \pm 1$ & $5.9 \pm 2.9$ \\
36 & $2 \pm 0.4$ & $2.4 \pm 0.6$ & $1.4 \pm 0.5$ & $1.5 \pm 0.5$ & $1.4 \pm 0.1$ & $1.4 \pm 1$ & $3.5 \pm 2.4$ & $1.6 \pm 1.5$ \\
39 & $1.1 \pm 0.3$ & $2.3 \pm 0.6$ & $0.5 \pm 0.3$ & $1.5 \pm 1.1$ & $1.7 \pm 0.3$ & $1.1 \pm 1.2$ & $1.1 \pm 0.5$ & $1.9 \pm 0.6$ \\
\hline
\end{tabular}

Data represent means from three replicates per treatment; CT-non-challenged control group; SB-synbiotic; SBST-synbiotic + S. typhimurium; ST-S. typhimurium; SBCP-synbiotic + C. perfringens; $\mathrm{CP}-\mathrm{C}$. perfringens; SBSTCP-synbiotic + S. typhimurium + C. perfringens; STCP-S. typhimurium + C. perfringens.

The morphometric index of the bursa allowed to determine the immunocompetence or immunosuppression in broilers between 7 and 42 days of age. When the index was 1.1 or higher was considered ideal, which translates into an immunocompetent organism. On the contrary, finding indexes equal to or lower than one would result in immunosuppression [28].

There were no broilers with immunosuppression signs in the synbiotic group according to the morphometric index. Meanwhile, immunosuppression was observed in the control group (2 birds) at day 39. At day 36, immunosuppressed broilers were found in the groups synbiotic + S. typhimurium (1 bird), C. perfringens (1 bird) and S. typhimurium + C. perfringens ( 2 birds). At day 39, birds with immunosuppression were found in most groups, except for the synbiotic and S. typhimurium + C. perfringens groups (Figure 1).

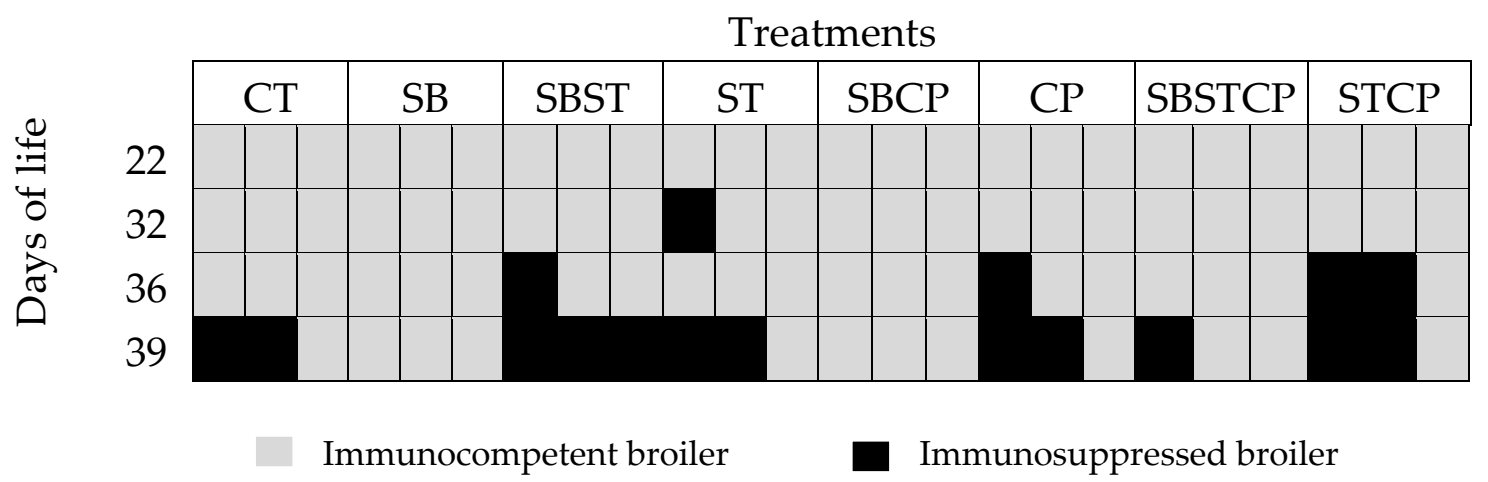

Figure 1. Bursa morphometric index in broilers treated with the synbiotic mix and inoculated with Salmonella typhimurium and Clostridium perfringens. CT-non-challenged control group; SB—synbiotic; SBST—synbiotic + S. typhimurium; ST—S. typhimurium; SBCP—synbiotic + C. perfringens; $\mathrm{CP}-\mathrm{C}$. perfringens; $\mathrm{SBSTCP}-$ synbiotic + S. typhimurium + C. perfringens; $\mathrm{STCP}-$ S. typhimurium + C. perfringens. Each square represents one broiler.

Histological analysis showed that the follicles of the synbiotic group were bigger $(p<0.05)$ in comparison to the control group at $32(363 \pm 111$ vs. $161 \pm 16 \mu \mathrm{m})$ and $39(304 \pm 78 \mathrm{vs} .180 \pm 32 \mu \mathrm{m})$ days of life. Likewise, the cortex of the synbiotic group had more volume in comparison to the control group $(p<0.05)$ at $32(196 \pm 78$ vs. $80 \pm 16 \mu \mathrm{m}), 36(236 \pm 67$ vs. $98 \pm 29 \mu \mathrm{m})$ and 39 (174 \pm 73 vs. $97 \pm 22 \mu \mathrm{m})$ days of life. Meanwhile, the medulla of the synbiotic group had more volume $(p<0.05)$ than the control group at $32(167 \pm 78$ vs. $82 \pm 23 \mu \mathrm{m})$ and $36(134 \pm 61$ vs. $78 \pm 22 \mu \mathrm{m})$ days of life 
(Supplementary Table S1). Larger follicles and cortex are indicative of better immunocompetence in broilers.

For broilers inoculated with S. typhimurium, those treated with the synbiotic (synbiotic + S. typhimurium) showed larger follicles when compare to those not treated at 32 (351 $\pm 54 \mathrm{vs}$. $203 \pm 63 \mu \mathrm{m})$ and 36 days of life $(420 \pm 38$ vs. $348 \pm 41 \mu \mathrm{m})$ (Supplementary Table S1).

In the lymphocyte counts per follicle of the bursa, it was observed that the follicles of the synbiotic group had a greater number of lymphocytes than the control $(p<0.05)$ at $22(109 \pm 14$ vs. $86 \pm 31)$ and 39 days (148 \pm 26 vs. $77 \pm 15$ ). For broilers inoculated with pathogens, the $S$. typhimurium group showed higher number of lymphocytes than the synbiotic + S. typhimurium group at day $22(105 \pm 18$ vs. $80 \pm 9)$ $(p<0.05)$; and in the groups inoculated with $C$. perfringens, those who were treated with the synbiotic (synbiotic $+C$. perfringens) had higher lymphocyte counts than those not treated (C. perfringens) at day 32 (180 \pm 24 vs. $91 \pm 24), 36$ (148 \pm 23 vs. $73 \pm 24)$ and day 39(154 \pm 20 vs. $96 \pm 23)$. In broilers treated with both pathogens, the synbiotic group + S. typhimurium + C. perfringens had more lymphocytes than S. typhimurium + C. perfringens $(p<0.05)$ at day $32(154 \pm 16$ vs. $126 \pm 24)($ Table 2$)$.

Table 2. Synbiotic effect on the number of lymphocytes per bursa follicle in broilers challenged with Salmonella typhimurium and Clostridium perfringens.

\begin{tabular}{|c|c|c|c|c|c|c|c|c|}
\hline \multirow{2}{*}{$\begin{array}{l}\text { Days of } \\
\text { Life }\end{array}$} & \multicolumn{8}{|c|}{ Lymphocytes } \\
\hline & CT & SB & SBST & ST & SBCP & $\mathrm{CP}$ & SBST CP & STCP \\
\hline 22 & $86 \pm 31^{a}$ & $109 \pm 14^{b}$ & $80 \pm 9^{A}$ & $105 \pm 18^{B}$ & $96 \pm 14$ & $80 \pm 22$ & $87 \pm 10^{\mathrm{A}}$ & $125 \pm 32^{B}$ \\
\hline 32 & $128 \pm 27$ & $138 \pm 30$ & $91 \pm 21$ & $92 \pm 20$ & $180 \pm 24^{a}$ & $91 \pm 24^{b}$ & $154 \pm 16^{\mathrm{A}}$ & $126 \pm 24^{B}$ \\
\hline 36 & $135 \pm 38^{a}$ & $103 \pm 13^{b}$ & $78 \pm 13$ & $85 \pm 22$ & $148 \pm 23^{a}$ & $73 \pm 24^{b}$ & $140 \pm 12$ & $152 \pm 27$ \\
\hline 39 & $77 \pm 15^{\mathrm{a}}$ & $148 \pm 26^{b}$ & $57 \pm 6$ & $76 \pm 22$ & $154 \pm 20^{a}$ & $96 \pm 23^{b}$ & $99 \pm 26$ & $87 \pm 21$ \\
\hline
\end{tabular}

CT: control group; SB: synbiotic mix; SBST: synbiotic mix + S. typhimurium; ST: S. typhimurium; SBCP: synbiotic mix + C. perfringens; CP: C. perfringens; SBSTCP: synbiotic mix + S. typhimurium + C. perfringens; STCP: S. typhimurium $+C$. perfringens. ${ }^{\mathrm{a}, \mathrm{b}, \mathrm{A}, \mathrm{B}}$ Values with different superscripts in the row are significantly different between adjacent columns $(p<0.05)$.

The histopathological analysis of the bursa showed signs of atrophy since day 22 of life in broilers' groups inoculated with the pathogens (S. typhimurium and S. typhimurium + C. perfringens). Intraepithelial and intrafolytic mucosal cysts, epithelial invaginations, epithelial folds in the folia, atrophy at the apex of the folds, and smaller lymphoid follicles were found. Broilers of the group synbiotic + S. typhimurium did not show histopathological lesions after 32 days (Figure 2). It should be noted that no apparent lesions were found on the bursa of broilers in the control, synbiotic, C. perfringens and synbiotic $+C$. perfringens groups.
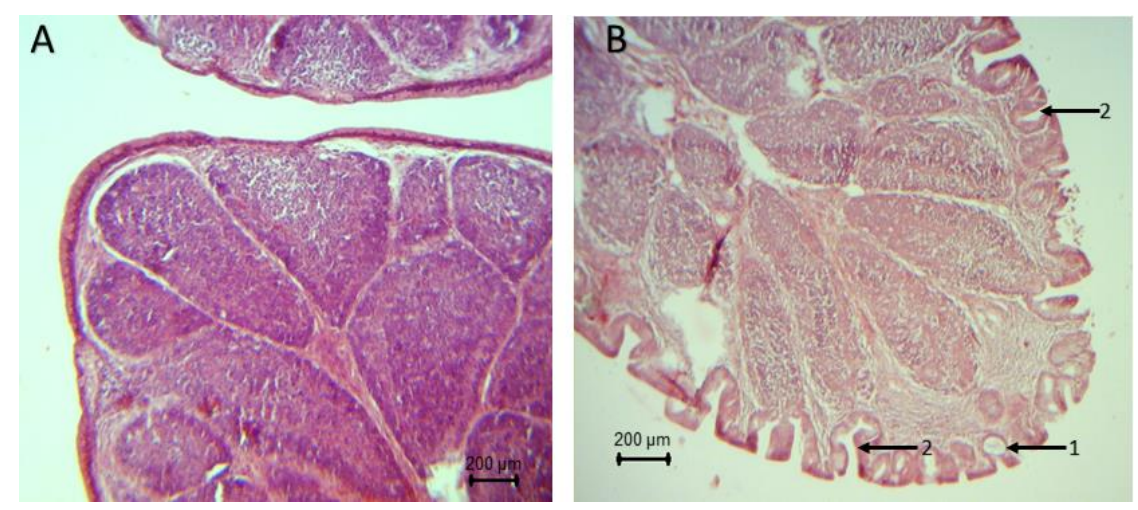

Figure 2. Histopathological lesions in the bursa of broilers challenged with S. typhimurium and C. perfringens. (A): Bursa without apparent lesions (control group) $(4 \times)$. (B): (1) intraepithelial mucosal cysts, (2) epithelial invaginations (S. typhimurium + C. perfringens) $(4 \times)$. 
The spleen weight was similar between treatments; at 22 days of life the average weight of the spleens was $1 \pm 0.1 \mathrm{~g}$, with a constant increase, until reaching an average weight of $2.3 \pm 0.4 \mathrm{~g}$ in all treatments at the end of the bioassay (39 days of life). The spleen of the broilers of the C. perfringens group was heavier $(p<0.05)$ at 36 days of life $(2.76 \pm 1.43$ vs. $1.60 \pm 0.10 \mathrm{~g})$, when compare to the synbiotic $+C$. perfringens group (Table 3$)$.

Table 3. Effect of dietary supplementation with a synbiotic mix on spleen weight of broilers orally inoculated with Salmonella typhimurium and Clostridium perfringens.

\begin{tabular}{|c|c|c|c|c|c|c|c|c|}
\hline \multirow{2}{*}{$\begin{array}{c}\text { Days of } \\
\text { Life }\end{array}$} & \multicolumn{8}{|c|}{ Spleen Weight } \\
\hline & $\mathrm{CT}$ & SB & SBST & ST & SBCP & $\mathrm{CP}$ & SBSTCP & STCP \\
\hline 22 & $0.9 \pm 0.1$ & $0.9 \pm 0.1$ & $1 \pm 0.1$ & $1 \pm 0.6$ & $1 \pm 0.1$ & $1 \pm 0.1$ & $1 \pm 0$ & $1 \pm 0.1$ \\
\hline 32 & $1.3 \pm 0.6$ & $1.3 \pm 0.6$ & $0.7 \pm 0.3$ & $1.3 \pm 0.6$ & $1.3 \pm 0.6$ & $1.3 \pm 0.6$ & $2 \pm 0$ & $1.3 \pm 0.6$ \\
\hline 36 & $1.4 \pm 0.2$ & $2.3 \pm 0.3$ & $1.8 \pm 0.6$ & $1.1 \pm 0.3$ & $1.6 \pm 0.1^{\mathrm{a}}$ & $2.8 \pm 1.4^{b}$ & $1.1 \pm 0.4$ & $1.5 \pm 0.2$ \\
\hline 39 & $1.8 \pm 1$ & $1.8 \pm 0.3$ & $2.2 \pm 0.6$ & $2.2 \pm 0.3$ & $2.5 \pm 0$ & $2.3 \pm 0.6$ & $2.5 \pm 0.5$ & $2.7 \pm 0.3$ \\
\hline
\end{tabular}

CT-non-challenged control group; SB-synbiotic; SBST-synbiotic + S. typhimurium; ST—S. typhimurium; $\mathrm{SBCP}-$ synbiotic $+C$. perfringens; $\mathrm{CP}-\mathrm{C}$. perfringens; $\mathrm{SBSTCP}-$ synbiotic $+S$. typhimurium $+C$. perfringens; STCP-S. typhimurium + C. perfringens. ${ }^{a, b}$ Values with different superscripts in the row are significantly different $(p<0.05)$.

The spleen morphometric index of the $C$. perfringens group showed a higher rate in comparison to the synbiotic $+C$. perfringens group at 36 days of life (1.5 \pm 1 vs. $08 \pm 0.1)$. No other differences were found between treatments (Table 4).

Table 4. Spleen morphometric index of broilers treated with the synbiotic mix and inoculated with Salmonella typhimurium and Clostridium perfringens.

\begin{tabular}{|c|c|c|c|c|c|c|c|c|}
\hline \multirow{2}{*}{$\begin{array}{c}\text { Days of } \\
\text { Life }\end{array}$} & \multicolumn{8}{|c|}{ Treatments } \\
\hline & CT & SB & SBST & ST & SBCP & $\mathrm{CP}$ & SBSTCP & STCP \\
\hline 22 & $1.2 \pm 0.1$ & $1 \pm 0.1$ & $1.3 \pm 0.3$ & $1.1 \pm 0$ & $1.1 \pm 0$ & $1.2 \pm 0$ & $1.2 \pm 0.1$ & $1.3 \pm 0.2$ \\
\hline 32 & $1.1 \pm 0.4$ & $1.1 \pm 0.4$ & $0.5 \pm 0.2$ & $1.8 \pm 0.8$ & $1 \pm 0.4$ & $1.1 \pm 0.5$ & $1.8 \pm 0.2$ & $1.3 \pm 0.9$ \\
\hline 36 & $0.8 \pm 0.1$ & $1.3 \pm 0$ & $1.1 \pm 0.3$ & $0.9 \pm 0.2$ & $0.8 \pm 0.1^{\mathrm{a}}$ & $1.5 \pm 1^{b}$ & $0.6 \pm 0.1$ & $0.8 \pm 0.1$ \\
\hline 39 & $0.8 \pm 0.5$ & $0.7 \pm 0.1$ & $0.8 \pm 0.2$ & $0.8 \pm 0$ & $85 \pm 22$ & $0.8 \pm 0.3$ & $1.1 \pm 0.2$ & $1 \pm 0.1$ \\
\hline
\end{tabular}

Data represent means from three replicates per treatment. CT-non-challenged control group; SB-synbiotic; $\mathrm{SBST}-$ synbiotic + S. typhimurium; ST—S. typhimurium; $\mathrm{SBCP}-$ synbiotic $+C$. perfringens; $\mathrm{CP}-\mathrm{C}$. perfringens; SBSTCP-synbiotic + S. typhimurium + C. perfringens; STCP-S. typhimurium $+C$. perfringens. ${ }^{a}, b$ Means with different superscripts within the same row differ significantly.

The white pulp lymphocyte count of the spleen was performed and at day 39 , the number of lymphocytes of the synbiotic group $(148 \pm 26)$ were higher $(p<0.05)$ than the control $(77 \pm 15)$. Regarding the birds inoculated with Salmonella, those treated with the synbiotic (synbiotic + S. typhimurium) showed lower numbers of lymphocytes when compared with the untreated (S. typhimurium) at 22 (3 2 vs. $17 \pm 7$ ) and 39 (576 vs. 7622 ) days; contrary to what happened with the broilers inoculated with $C$. perfringens, where those treated with the synbiotic (synbiotic + C. perfringens) had a higher number of lymphocytes at day $39(154 \pm 20)$ compared to the untreated (C. perfringens) group (96 \pm 23$)$ (Table 5).

There were no differences in the number of lymphocytes between the synbiotic $+S$. typhimurium + C. perfringens and S. typhimurium + C. perfringens groups (Table 5).

The qualitative histopathological analysis showed discrete to moderate lymphoid depletion in the spleen of broilers inoculated with pathogens (S. typhimurium, C. perfringens and S. typhimurium + C. perfringens groups) from 22 days of age until the end of the bioassay. However, with the synbiotic and the pathogens (synbiotic mix $+S$. typhimurium, synbiotic mix $+C$. perfringens and synbiotic mix + S. typhimurium + C. perfringens), no signs of lymphoid depletion at day 39 were shown, also, no histopathological injuries were identified in the control and synbiotic groups (Figure 3). 
Table 5. Synbiotic effect on the white pulp lymphocyte count of broilers' spleens challenged with Salmonella typhimurium and Clostridium perfringens.

\begin{tabular}{ccccccccc}
\hline $\begin{array}{c}\text { Days of } \\
\text { Life }\end{array}$ & Lymphocytes \\
\cline { 2 - 8 } & CT & SB & SBST & ST & SBCP & CP & SBSTCP & STCP \\
\hline 22 & $8 \pm 3$ & $7 \pm 9$ & $3 \pm 2^{\mathrm{A}}$ & $17 \pm 7^{\mathrm{B}}$ & $9 \pm 4$ & $9 \pm 7$ & $12 \pm 9$ & $9 \pm 3$ \\
32 & $3 \pm 1$ & $3 \pm 2$ & $6 \pm 4$ & $10 \pm 4$ & $11 \pm 5$ & $14 \pm 8$ & $17 \pm 10$ & $15 \pm 7$ \\
36 & $5 \pm 5$ & $5 \pm 5$ & $5 \pm 4$ & $9 \pm 4$ & $12 \pm 5$ & $17 \pm 5$ & $8 \pm 6$ & $7 \pm 3$ \\
39 & $77^{\mathrm{a}} \pm 15^{\mathrm{a}}$ & $148 \pm 26^{\mathrm{b}}$ & $57 \pm 6^{\mathrm{A}}$ & $76 \pm 22^{\mathrm{B}}$ & $154 \pm 20^{\mathrm{a}}$ & $96 \pm 23^{\mathrm{b}}$ & $99 \pm 26$ & $87 \pm 21$ \\
\hline
\end{tabular}

CT: control group; SB: synbiotic mix; SBST: synbiotic mix + S. typhimurium; ST: S. typhimurium; SBCP: synbiotic mix + C. perfringens; CP: C. perfringens; SBSTCP: synbiotic mix + S. typhimurium + C. perfringens; STCP: S. typhimurium $+C$. perfringens. ${ }^{a, b}, \mathrm{~A}, \mathrm{~B}$ Values with different superscripts in the row are significantly different between adjacent columns $(p<0.05)$.
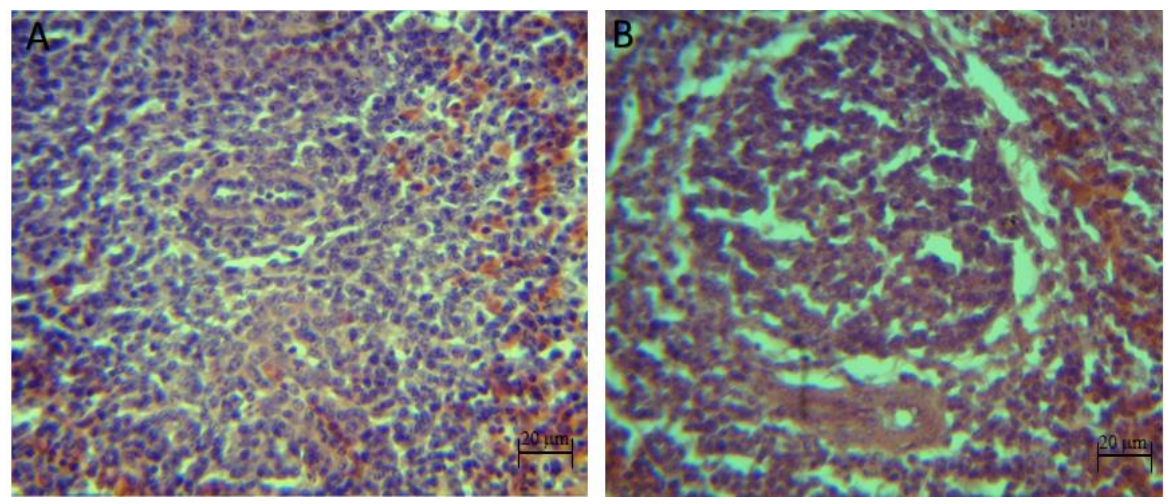

Figure 3. Histopathological injuries in the spleen of broilers chickens challenged with S. typhimurium and C. perfringens. (A): Spleen without apparent lesions (control group) (40×). (B): Lymphoid depletion in the spleen (S. typhimurium + C. perfringens group) $(40 \times)$.

Histological analysis of thymus showed that the follicles of the control group were smaller $(p<0.05)$ when compared to the synbiotic group at $22(489 \pm 151$ vs. $803 \pm 251 \mu \mathrm{m}), 36(474 \pm 165 \mathrm{vs}$. $995 \pm 359 \mu \mathrm{m})$ and $39(410 \pm 149$ vs. $987 \pm 195 \mu \mathrm{m})$ days of age.

At 22 days of life, broilers of $C$. perfringens group had bigger follicles $(1323 \pm 331 \mu \mathrm{m})$ in comparison to synbiotic $+C$. perfringens group $(918 \pm 348 \mu \mathrm{m})(p<0.05)$. Thymus follicles of the birds from the $S$. typhimurium group were smaller $(p<0.05)$ at day $36(554 \pm 270 \mu \mathrm{m})$ than the synbiotic + S. typhimurium group $(1023 \pm 402 \mu \mathrm{m})$. The cortex of the thymus follicles of the synbiotic group had more volume when compare to the control group $(p<0.05)$ at $22(497 \pm 173$ vs. $292 \pm 86 \mu \mathrm{m}), 36$ $(610 \pm 238$ vs. $290 \pm 138 \mu \mathrm{m})$ and 39 (565 \pm 195 vs. $248 \pm 107 \mu \mathrm{m})$ days of life.

At 22 days of life, broilers of the $C$. perfringens group had cortexes with more volume (796 $\pm 234 \mu \mathrm{m})$ compare to the synbiotic $+C$. perfringens group $(532 \pm 199 \mu \mathrm{m})(p<0.05)$. Thymus follicles cortexes of the birds of the $S$. typhimurium group were smaller $(p<0.05)$ at day $36(343 \pm 135 \mu \mathrm{m})$ when compare to the synbiotic $+S$. typhimurium group $(670 \pm 213 \mu \mathrm{m})$. The evaluation of the thymus medulla showed that it had a higher volume in the synbiotic group when compared to control the group at 36 $(385 \pm 236$ vs. $183 \pm 69 \mu \mathrm{m})$ and $39(423 \pm 128$ vs. $163 \pm 65 \mu \mathrm{m})$ days. The same situation was observed in the $C$. perfringens group, who had bigger medulla $(p<0.05)$ when compared to the synbiotic + C. perfringens group at $22(527 \pm 165$ vs. $387 \pm 176 \mu \mathrm{m})$ and $32(628 \pm 361 \mathrm{vs} .417 \pm 173 \mu \mathrm{m})$ days of life (Supplementary Table S2).

Concerning the number of lymphocytes per thymus follicle, the control group had fewer lymphocytes than the synbiotic group $(p<0.05)$ at $22(37 \pm 14$ vs. $135 \pm 50)$ and 32 days $(83 \pm 13$ vs. $239 \pm 78$ ). In broilers challenged with $C$. perfringens those who were treated with the synbiotic (synbiotic + C. perfringens group) had a higher number of lymphocytes at $36(348 \pm 28$ vs. $284 \pm 52)$ and 39 days ( $390 \pm 43$ vs. $284 \pm 53$ ) when compare to those not treated (C. perfringens). In broilers challenged with both pathogens, those who were treated with the synbiotic (synbiotic mix + S. typhimurium + 
C. perfringens) had a higher number of lymphocytes at day 22 compared to those not treated ( $298 \pm 43$ vs. $193 \pm 41$ ). However, this was modified at the end of the bioassay (39 days of life), where the broilers of the $C$. perfringens group showed higher counts of lymphocytes than those of the synbiotic + C. perfringens group (337 \pm 38 vs. $256 \pm 43)$ (Table 6).

Table 6. Synbiotic effect on the count of lymphocytes per thymus follicle in broilers challenged with Salmonella typhimurium and Clostridium perfringens in different days of life.

\begin{tabular}{|c|c|c|c|c|c|c|c|c|}
\hline $\begin{array}{c}\text { Days of } \\
\text { Life }\end{array}$ & \multicolumn{8}{|c|}{ Lymphocytes } \\
\hline 22 & $37 \pm 14^{\mathrm{A}}$ & $135 \pm 50^{B}$ & $147 \pm 52^{a}$ & $93 \pm 28^{b}$ & $150 \pm 34$ & $172 \pm 40$ & $298 \pm 43^{a}$ & $193 \pm 41^{b}$ \\
\hline 32 & $83 \pm 13^{A}$ & $239 \pm 78^{B}$ & $204 \pm 17$ & $204 \pm 46$ & $278 \pm 36$ & $287 \pm 50$ & $275 \pm 23$ & $270 \pm 49$ \\
\hline 36 & $155 \pm 32$ & $151 \pm 22$ & $200 \pm 30$ & $167 \pm 29$ & $348 \pm 28^{A}$ & $284 \pm 52^{B}$ & $260 \pm 58$ & $263 \pm 47$ \\
\hline 39 & $126 \pm 24$ & $150 \pm 21$ & $186 \pm 35$ & $213 \pm 49$ & $390 \pm 43^{\mathrm{A}}$ & $284 \pm 53^{B}$ & $256 \pm 43^{a}$ & $337 \pm 38^{b}$ \\
\hline
\end{tabular}

CT: control group; SB: synbiotic mix; SBST: synbiotic mix + S. typhimurium; ST: S. typhimurium; SBCP: synbiotic mix + C. perfringens; CP: C. perfringens; SBSTCP: synbiotic mix + S. typhimurium + C. perfringens; STCP: S. typhimurium $+C$. perfringens. ${ }^{a, b, A}, \mathrm{~B}$ Values with different superscripts in the row are significantly different between adjacent columns $(p<0.05)$

In the histopathological analysis, no apparent injuries were found on the thymus of the control and synbiotic groups during the bioassay. Groups challenged with the pathogens (S. typhimurium, C. perfringens and S. typhimurium + C. perfringens) showed discrete to moderate infiltration of heterophils, degeneration and necrosis of reticular epithelial cells in the medulla at 22, 32, 36 and 39 days of life. The groups treated with the synbiotic (synbiotic + S. typhimurium, synbiotic $+C$. perfringens and synbiotic $+S$. typhimurium + C. perfringens) showed those injuries at 22 and 32 days of life (Figure 4).

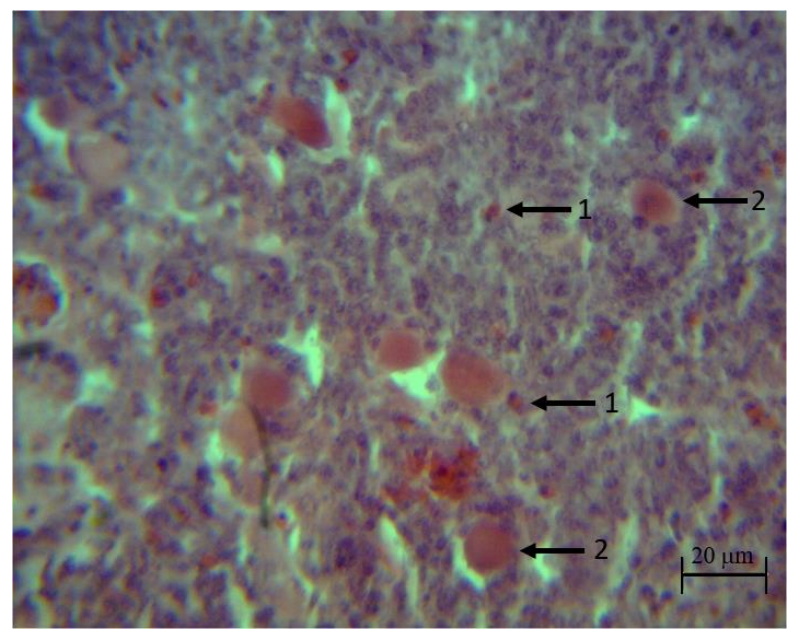

Figure 4. Histopathological injuries in the thymus of broilers challenged with S. typhimurium and C. perfringens $(100 \times)$. (1) heterophiles (2) reticular epithelial cells (S. typhimurium + C. perfringens)

The IgA concentration of the control group was higher than the synbiotic group at day 22 $(78.7 \pm 3.2$ vs. $67.5 \pm 0.6)(p<0.05)$, however, this situation changed at day 36 when the synbiotic group showed a higher IgA concentration $(p<0.05)$ when compared to the control $(164.4 \pm 1.5 \mathrm{vs}$. $151.7 \pm 2.3)$. In groups infected with $S$. typhimurium, the IgA concentration was significantly higher in the synbiotic $+S$. typhimurium group in all samples $(p<0.05)$; similar results were found in the chickens infected with $C$. perfringens, where the IgA concentration of the synbiotic $+C$. perfringens group was higher than those of the $C$. perfringens group in most samples $(p<0.05)$. A higher IgA concentration was found in the synbiotic $+S$. typhimurium $+C$. perfringens group when compared to its control, at all times except the last sampling (day 39) $(146.4 \pm 1.1$ vs. $84.9 \pm 2.6)(p<0.05)$ (Table 7). 
Table 7. Synbiotic effect on serum IgA concentration in broilers challenged with Salmonella typhimurium and Clostridium perfringens (ng $\mathrm{mL}^{-1}$ ) in different days of life.

\begin{tabular}{ccccc}
\hline \multirow{2}{*}{ Treatments } & \multicolumn{4}{c}{$\mathbf{n g ~ m L}^{-\mathbf{1}}$} \\
\cline { 2 - 5 } & 22 days & 32 days & 36 days & 39 days \\
\hline CT & $78.7 \pm 3.2$ & $117 \pm 1.8^{\mathrm{a}}$ & $151.7 \pm 2.3$ & $156 \pm 2.8^{\mathrm{a}}$ \\
SB & $67.5 \pm 0.6$ & $112.4 \pm 3.9^{\mathrm{a}}$ & $164.4 \pm 1.5$ & $156.5 \pm 1.7^{\mathrm{a}}$ \\
SBST & $79.5 \pm 2.3$ & $222.1 \pm 1.5$ & $196.9 \pm 1.9$ & $166.2 \pm 1.6$ \\
ST & $59 \pm 0.2$ & $146.4 \pm 2.3$ & $171.2 \pm 1.3$ & $77.2 \pm 3.6$ \\
SBCP & $45.2 \pm 0.7$ & $182.8 \pm 1.1$ & $119.1 \pm 0.1^{\mathrm{a}}$ & $101.9 \pm 1.4$ \\
CP & $37 \pm 0.4$ & $169.3 \pm 2.9$ & $125.6 \pm 1.7^{\mathrm{a}}$ & $87.67 \pm 5.4$ \\
SBSTCP & $83 \pm 2.6$ & $149.2 \pm 0.7$ & $217.87 \pm 1.5$ & $84.9 \pm 2.6$ \\
STCP & $66.5 \pm 2.6$ & $161 \pm 1.1$ & $205.8 \pm 6$ & $146.4 \pm 1.1$ \\
\hline
\end{tabular}

CT: control group; SB: synbiotic mix; SBST: synbiotic mix + S. typhimurium; ST: S. typhimurium; SBCP: synbiotic mix + C. perfringens; CP: C. perfringens; SBSTCP: synbiotic mix + S. typhimurium + C. perfringens; STCP: S. typhimurium + C. perfringens. ${ }^{a}$ Means with superscripts in the same column are homogeneous $(p<0.05)$.

\section{Discussion}

Bursa, thymus and spleen are the producers of immune cells, orientation of the cells occurs more efficiently in healthy animals than in immune-compromised ones, higher relative weights of lymphoid organs could be the consequence of amplified B and T lymphocytes. In disease-free animals, an increase in weight of immune organs correlates with enhanced proliferation of immune cells, which represents better immunity of the body [29].

In assessing the bursa weight, the synbiotic group was identified as the only group that showed sustained bursa growth throughout the bioassay, the variation of the weight of the bursa in the other groups may be the result of stress caused by pathogenic microorganisms inoculated to birds. According to the anatomic-physiological development of the bursa of Fabricius in normal conditions and free of stress, it is expected that this organ maintains a sustained growth until broilers reach eight weeks of life, where it will naturally begin the process of atrophy. Several authors have reported moderate atrophy of the bursa towards the sixth or seventh week in birds subjected to different challenges such as bedding reuse, Gumboro disease infection [30,31], immobilization, turning [19], heat stress [32] and overcrowding [33]. Decreased bursa weight is known to be associated with immunosuppression, Gomes et al., [33] found that by decreasing bursa weight, broilers are more susceptible to enteritis caused by Salmonella enterica subsp. enterica serovar Enteritidis.

In the spleen weight of the broilers treated with the synbiotic no differences were found. Similar results were reported by Alkhalf et al. (2010), who found no difference in the spleen weight of broilers treated with a probiotic. This may indicate that the spleen develops its proper functions with age [34]. However, it has been reported an increase in the size and weight of chickens' spleens treated with probiotics and synbiotics [35,36].

Although the morphometric index of the bursa of broilers inoculated with the pathogenic microorganisms (S. typhimurium, C. perfringens and S. typhimurium + C. perfringens) did not indicate immunosuppression, the histopathological analysis of the organs revealed signs of atrophy at day 22; changes in the integrity as well as in the function of the bursa can cause changes in the production of immunoglobulins [33] which implies immunosuppression. These results indicate that macroscopic evaluation of the bursa does not always reflect the immune capacity or damage degree of the bursa [30]. It should be noted that no signs of atrophy were found in the histopathological analysis of the synbiotic group, which is consistent with the morphometric index (equal to or greater than 1.1) found in that group.

The development of lymphoid tissues can reflect the immune system response and functionality. Bursa of Fabricius, spleen and thymus are the main immune organs involved in humoral and cell immunity of animals [37]. Bursa of Fabricius is required for B-cell development, the function of spleen, 
the biggest peripheral immune organ, is involved in the chicken's immune reaction, and thymus is the site of T-cell maturation [38,39].

In this study, the follicles from the bursa of broilers treated with the synbiotic, inoculated or not with pathogens, showed a significant cortex increase $(p<0.05)$ when compared to those who were not treated. When evaluating the thymus, this tendency only occurred between the synbiotic and control groups, where the cortex of the synbiotic group's follicles was thicker $(p<0.05)$ than those of the control group. The histomorphological variations in the cortex and medulla of bursa and thymus, have a link with immune function. A larger cortex could be a sign of the rapid maturation rate of thymocytes [29] which can be proven by noting that those follicles with a larger cortex also had a higher lymphocyte count when compared to those with a smaller cortex, also it has been reported that specific probiotics like B. infantis, B. animalis [40], L. acidophilus, L. reuteri, L. salivarius [41] and L. rhamnosus [42] have been shown to induce an increase in lymphocytes B and $T$.

The spleen evaluation showed a lymphoid depletion in broilers challenged with $S$. typhimurium and C. perfringens, as published by Parsons et al., this depletion is part of the histopathological changes caused by $S$. typhimurium [43].

The histopathological lesions found in the bursa of the challenged broilers are associated to Salmonella infection. Loss of lymphoid tissue and degeneration of the bursa, has been reported by Garcia et al., Kumari et al. and Lopes et al., who suggested that the lesions of the bursa could have resulted from adverse physiological conditions related with Salmonella infection, like anorexia, dehydration, anemia, etc. [44-46]. It should be noted that in broilers challenged with S. typhimurium, but treated with the synbiotic, the lesions occurred only at 22 and 32 days of life and showed less intensity when compared to untreated broilers.

No apparent lesions were found in the bursa of chickens challenged with $C$. perfringens in this work, although the presence of $C$. perfringens is associated with an increased risk of infectious bursal disease [47]; similar results were reported by Ao et al [48].

In general, broilers treated with the synbiotic, challenged or not with the pathogens, had higher IgA concentration than untreated ones. It was reported that IgA production can be induced by the administration of probiotics. Sakai et al. [49], found that oral administration of Lactobacillus gasseri LG2055 induced IgA production and increased the rate of IgA+ cell population in Peyer's patch and in the lamina propria. Similar results were reported by Gao et al., when administered L. plantarum strain IMAU10120 (LP-8) [50]

IgA plays an important role in host defense against mucosal transmitted pathogens, prevents commensal bacteria from binding to epithelial cells and neutralizes their toxins to maintain homeostasis at the mucosal surfaces, also, secretion of IgA is critical in the regulation of the composition of the microbial community in the gut [40]

\section{Conclusions}

The synbiotic composed of Lactobacillus rhamnosus HN001, Pediococcus acidilactici MA18/5 M and Agave tequilana fructans can stimulate the bursa and the IgA production, increasing the size of its follicles and promoting the ability to resist infections caused by $S$. typhimurium in broilers.

Supplementary Materials: The following are available online at http://www.mdpi.com/2076-2615/10/5/886/s1, Table S1: Morphology of the bursa of broilers treated with the synbiotic mix and inoculated with Salmonella typhimurium and Clostridium perfringens, Table S2: Morphology of the thymus of broilers treated with the synbiotic mix and inoculated with Salmonella typhimurium and Clostridium perfringens.

Author Contributions: Conceptualization A.V.-L.; data curation, Z.V.-d.1.M.; formal analysis, Z.V.-d.1.M. and O.V.-P.; funding acquisition, A.V.-L.; investigation, Z.V.-d.l.M., K.N., O.V.-P., H.A. and A.V.-L.; methodology, K.N. and A.V.-L.; project administration, A.V.-L.; resources, A.V.-L.; supervision, K.N., O.V.-P., F.A. and A.V.-L.; writing—original draft, Z.V.-d.l.M.; writing—review and editing, K.N., F.A. and A.V.-L. All authors have read and agreed to the published version of the manuscript. 
Funding: This work is part of the project 'synbiotic avian vector for the improvement of feed efficiency and prevention of infections.' Project number 220311. Funded under the call for proposals PEI/CONACyT 2015 and by the company Kurago Biotek. Zuamí Villagrán-de la Mora holds a doctorate scholarship from the Teacher Professional Development Program (PRODEP).

Acknowledgments: We are grateful for the support given by the histology laboratory of CUCBA and its staff: Victor Barragan Cano and Xóchitl Rocío Ávila Dávila.

Conflicts of Interest: The authors declare no conflicts of interest.

\section{References}

1. Kabploy, K.; Bunyapraphatsara, N.; Morales, N.P.; Paraksa, N. Effect of antibiotic growth promoters on anti-oxidative and anti-inflammatory activities in broiler chickens. Thai J. Vet. Med. 2016, 46, 89-95.

2. Erdoĝan, Z.; Erdoĝan, S.; Aslantaş, Ö.; Çelik, S. Effects of dietary supplementation of synbiotics and phytobiotics on performance, caecal coliform population and some oxidant/antioxidant parameters of broilers. J. Anim. Physiol. Anim. Nutr. 2010, 94, e40-e48. [CrossRef] [PubMed]

3. Naghi, A.S.; Ghasemi, H.A.; Taherpour, K. Evaluation of Aloe vera and synbiotic as antibiotic growth promoter substitutions on performance, gut morphology, immune responses and blood constitutes of broiler chickens. Anim. Sci. J. 2017, 88, 306-313. [CrossRef] [PubMed]

4. Van Immerseel, F.; De Buck, J.; Pasmans, F.; Huyghebaert, G.; Haesebrouck, F.; Ducatelle, R. Clostridium perfringens in poultry: An emerging threat for animal and public health. Avian Pathol. 2004, 33, 537-549. [CrossRef] [PubMed]

5. Min, Y.N.; Yang, H.L.; Xu, Y.X.; Gao, Y.P. Effects of dietary supplementation of synbiotics on growth performance, intestinal morphology, sIgA content and antioxidant capacities of broilers. J. Anim. Physiol. Anim. Nutr. 2016, 100, 1073-1080. [CrossRef] [PubMed]

6. Talebi, A.; Amani, A.; Pourmahmod, M.; Saghaei, P.; Rezaie, R. Synbiotic enhances immune responses against infectious bronchitis, infectious bursal disease, Newcastle disease and avian influenza in broiler chickens. Vet. Res. Forum 2015, 6, 191-197.

7. Huyghebaert, G.; Ducatelle, R.; Van Immerseel, F. Van An update on alternatives to antimicrobial growth promoters for broilers. Vet. J. 2011, 187, 182-188. [CrossRef]

8. Akpabio, U. Epidemiology of Poultry Salmonellosis: A Review. J. Vet. Adv. 2015, 5, 902. [CrossRef]

9. Chen, H.M.; Wang, Y.; Su, L.H.; Chiu, C.H. Nontyphoid Salmonella infection: Microbiology, clinical features, and antimicrobial therapy. Pediatr. Neonatol. 2013, 54, 147-152. [CrossRef]

10. Sohail, M.U.; Ijaz, A.; Younus, M.; Shabbir, M.Z.; Kamran, Z.; Ahmad, S.; Anwar, H.; Yousaf, M.S.; Ashraf, K.; Shahzad, A.H.; et al. Effect of supplementation of mannan oligosaccharide and probiotic on growth performance, relative weights of viscera, and population of selected intestinal bacteria in cyclic heat-stressed broilers. J. Appl. Poult. Res. 2013, 22, 485-491. [CrossRef]

11. Ghasemi, H.A.; Kasani, N.; Taherpour, K. Effects of black cumin seed (Nigella sativa L.), a probiotic, a prebiotic and a synbiotic on growth performance, immune response and blood characteristics of male broilers. Livest. Sci. 2014, 164, 128-134. [CrossRef]

12. Casteleyn, C.; Doom, M.; Lambrechts, E.; Van den Broeck, W.; Simoens, P.; Cornillie, P. Locations of gut-associated lymphoid tissue in the 3-month-old chicken: A review. Avian Pathol. 2010, 39, 143-150. [CrossRef] [PubMed]

13. Korver, D.R. Implications of changing immune function through nutrition in poultry. Anim. Feed Sci. Technol. 2012, 173, 54-64. [CrossRef]

14. Adil, S.; Magray, S.N. Impact and manipulation of gut microflora in poultry: A review. J. Anim. Vet. Adv. 2012, 6, 873-877. [CrossRef]

15. Khan, R.U.; Naz, S. The applications of probiotics in poultry production. Worlds. Poult. Sci. J. 2013, 69, 621-632. [CrossRef]

16. Lammers, K.M.; Brigidi, P.; Vitali, B.; Gionchetti, P.; Rizzello, F.; Caramelli, E.; Matteuzzi, D.; Campieri, M. Immunomodulatory effects of probiotic bacteria DNA: IL-1 and IL-10 response in human peripheral blood mononuclear cells. FEMS Immunol. Med. Microbiol. 2003, 38, 165-172. [CrossRef]

17. Yan, F.; Polk, D.B. Lactobacillus rhamnosus GG: An Updated Strategy to Use Microbial Products to Promote Health. Funct. Food Rev. 2012, 4, 77-84. [CrossRef] 
18. Sandoval, G.; Terraes, J.; Revidatti, F.; Fernandez, R.; Merlo, W.A.; Esquivel, G.P.; Zbinden, C. Efecto de una Maniobra de Inmovilización e Inversión Corporal Sobre la Función Metabólica del Hígado en Pollos Parrilleros. Available online: https:/www.researchgate.net/publication/268429078_Efecto_de_una_maniobra_de_ inmovilizacion_e_inversion_corporal_sobre_la_funcion_metabolica_del_higado_en_pollos_parrilleros (accessed on 14 January 2019).

19. Revidatti, F.A.; Fernández, R.J.; Terraes, J.C.; Sandoval, G.L.; Esquivel de Luchi, P. Modificaciones del peso corporal e indicadores de estrés en pollos parrilleros sometidos a inmovilización y volteo. Rev. Vet. 2002, 12, $1 \mathrm{y} 2$.

20. Villagran-de la Mora, Z.; Nuño, K.; Olga, V.; Avalos, H.; Castro-rosas, J.; Carlos, G.; Angulo, C.; Ascencio, F. Effect of a Synbiotic Mix on Intestinal Structural Changes, and Salmonella Typhimurium and Clostridium Perfringens Colonization in Broiler Chickens. Animals 2019, 9, 777. [CrossRef]

21. Cooper, K.K.; Songer, J.G. Necrotic enteritis in chickens: A paradigm of enteric infection by Clostridium perfringens type A. Anaerobe 2009, 15, 55-60. [CrossRef]

22. La Ragione, R.M.; Narbad, A.; Gasson, M.J.; Woodward, M.J. In vivo characterization of Lactobacillus johnsonii FI9785 for use as a defined competitive exclusion agent against bacterial pathogens in poultry. Lett. Appl. Microbiol. 2004, 38, 197-205. [CrossRef] [PubMed]

23. Garden, M.; Singleton, R. Manejo del Pollo de Engorde para un Peso Liviano al Mercado (de 1.5 a $1.8 \mathrm{Kg} / \mathrm{de}$ 3.3 a 4.0 lb). Arbor Acres Boletín Serv. 2008, 4, 1-7.

24. Valladares, J. Necropsias en Aves | BM Editores. Available online: http://www.produccion-animal.com.ar/ produccion_aves/enfermedades_aves/30-Necropsias_en_Aves.pdf (accessed on 13 March 2019).

25. ISBER 2012 Best Practices for Repositories Collection, Storage, Retrieval, and Distribution of Biological Materials for Research International Society for Biological and Environmental Repositories. Biopreserv. Biobank. 2012, 10, 79-161. [CrossRef] [PubMed]

26. Tambini, A.; Alba, M.; Perales, R.; Falcón, N. Evaluación Anátomo-Histopatológica de bursa, timo y bazo de pollos de carnecriados sobre cama reutilizada vs. cama neva. Rev. Investig. Vet. Perú 2010, 21, 180-186.

27. Muniz, E.C.; Fascina, V.B.; Pires, P.P.; Carrijo, A.S.; Guimarães, E.B. Histomorphology of Bursa of Fabricius: Effects of Stock Densities on Commercial Broilers. Braz. J. Poult. Sci. 2006, 8, 217-220. [CrossRef]

28. Cazaban, C.; Masferrer, N.M.; Pascual, R.D.; Espadamala, M.N.; Costa, T.; Gardin, Y. Proposed bursa of fabricius weight to body weight ratio standard in commercial broilers. Poult. Sci. 2015, 94, 2088-2093. [CrossRef]

29. Sikandar, A.; Zaneb, H.; Younus, M.; Masood, S.; Aslam, A.; Shah, M.; Rehman, H. Growth performance, immune status and organ morphometry in broilers fed bacillus subtilis-supplemented diet. S. Afr. J. Anim. Sci. 2017, 47, 378-388. [CrossRef]

30. Aihara, N.; Horiuchi, N.; Hikichi, N.; Ochiai, M.; Hosoda, Y.; Ishikawa, Y.; Shimazaki, Y.; Oishi, K. Immunoreactivity and morphological changes of bursal follicles in chickens infected with vaccine or wild-type strains of the infectious bursal disease virus. J. Vet. Med. Sci. 2015, 77, 913-918. [CrossRef]

31. Zhao, S.; Jia, Y.; Han, D.; Ma, H.; Zahid, S.; Shah, A.; Ma, Y. Influence of the structural development of bursa on the susceptibility of chickens to infectious bursal disease virus. Poult. Sci. 2016, 95, 2786-2794. [CrossRef]

32. Bartlett, J.R.; Smith, M.O. Effects of Different Levels of Zinc on the Performance and Immunocompetence of Broilers Under Heat Stress. Poult. Sci. 2003, 82, 1580-1588. [CrossRef]

33. Gomes, A.V.S.; Quinteiro-Filho, W.M.; Ribeiro, A.; Ferraz-de-Paula, V.; Pinheiro, M.L.; Baskeville, E.; Akamine, A.T.; Astolfi-Ferreira, C.S.; Ferreira, A.J.P.; Palermo-Neto, J. Overcrowding stress decreases macrophage activity and increases Salmonella Enteritidis invasion in broiler chickens. Avian Pathol. 2014, 43, 82-90. [CrossRef] [PubMed]

34. Alkhalf, A.; Alhaj, M.; Al-Homidan, I. Influence of Probiotic Supplementation on Immune Response of Broiler Chicks. Egypt. Poult. Sci. 2010, 30, 271-280.

35. Madej, J.P.; Bednarczyk, M. Effect of in ovo- delivered prebiotics and synbiotics on the morphology and specific immune cell composition in the gut-associated lymphoid tissue. Poult. Sci. 2016, 95, 19-29. [CrossRef] [PubMed]

36. Awad, W.A.; Ghareeb, K.; Abdel-Raheem, S.; Bohm, J. Effects of dietary inclusion of probiotic and synbiotic on growth performance, organ weights, and intestinal histomorphology of broiler chickens. Poult. Sci. 2009, 88, 49-56. [CrossRef] [PubMed] 
37. Zhang, T.; Xie, J.; Zhang, M.; Fu, N.; Zhang, Y. Effect of a potential probiotics Lactococcus garvieae B301 on the growth performance, immune parameters and caecum microflora of broiler chickens. J. Anim. Physiol. Anim. Nutr. 2016, 100, 413-421. [CrossRef] [PubMed]

38. Ciriaco, E.; Píñera, P.P.; Díaz-Esnal, B.; Laurà, R. Age-Related Changes in the Avian Primary Lymphoid Organs (Thymus and Bursa of Fabricius). Microsc. Res. Tech. 2003, 62, 482-487. [CrossRef]

39. Gao, P.; Ma, C.; Sun, Z.; Wang, L.; Huang, S.; Su, X.; Xu, J.; Zhang, H. Feed-additive probiotics accelerate yet antibiotics delay intestinal microbiota maturation in broiler chicken. Microbiome 2017, 5, 91. [CrossRef]

40. Frei, R.; Akdis, M.; O'mahony, L. Prebiotics, probiotics, synbiotics, and the immune system: Experimental data and clinical evidence. Curr. Opin. Gastroenterol. 2015, 31, 153-158. [CrossRef]

41. Brisbin, J.T.; Gong, J.; Orouji, S.; Esufali, J.; Mallick, A.I.; Parvizi, P.; Shewen, P.E.; Sharif, S. Oral Treatment of Chickens with Lactobacilli Influences Elicitation of Immune Responses. Clin. Vaccine Immunol. 2011, 18, 1447-1455. [CrossRef]

42. Haghighi, H.R.; Gong, J.; Gyles, C.L.; Hayes, M.A.; Sanei, B.; Parvizi, P.; Gisavi, H.; Chambers, J.R.; Sharif, S. Modulation of antibody-mediated immune response by probiotics in chickens. Clin. Diagn. Lab. Immunol. 2005, 12, 1387-1392. [CrossRef]

43. Parsons, B.N.; Humphrey, S.; Salisbury, A.M.; Mikoleit, J.; Hinton, J.C.D.; Gordon, M.A.; Wigley, P. Invasive Non-Typhoidal Salmonella Typhimurium ST313 Are Not Host-Restricted and Have an Invasive Phenotype in Experimentally Infected Chickens. PLoS Negl. Trop. Dis. 2013, 7, 1-8. [CrossRef] [PubMed]

44. Garcia, K.O.; Santana, A.M.; Neto, O.C.F.; Simplício, K.M.M.G.; Alessi, A.C.; Júnior, Â.B.; Fagliari, J.J. Experimental infection of commercial layers using a Salmonella enterica sorovar Gallinarum strain: Blood serum components and histopathological changes. Braz. J. Vet. Pathol. 2010, 3, 111-117.

45. Kumari, D.; Mishra, S.K.; Lather, D. Pathomicrobial studies on Salmonella gallinarum infection in broiler chickens. Vet. World 2013, 6, 725-729. [CrossRef]

46. Lopes, P.D.; Freitas Neto, O.C.; Batista, D.F.A.; Denadai, J.; Alarcon, M.F.F.; Almeida, A.M.; Vasconcelos, R.O.; Setta, A.; Barrow, P.A.; Berchieri, A. Experimental infection of chickens by a flagellated motile strain of Salmonella enterica serovar Gallinarum biovar Gallinarum. Vet. J. 2016, 214, 40-46. [CrossRef]

47. Mahgoub, H.A. An overview of infectious bursal disease. Arch. Virol. 2012, 157, 2047-2057. [CrossRef]

48. Ao, Z.; Kocher, A.; Choct, M. Effects of dietary additives and early feeding on performance, gut development and immune status of broiler chickens challenged with clostridium perfringens. Asian Australas. J. Anim. Sci. 2012, 25, 541-551. [CrossRef]

49. Sakai, F.; Hosoya, T.; Ono-Ohmachi, A.; Ukibe, K.; Ogawa, A.; Moriya, T.; Kadooka, Y.; Shiozaki, T.; Nakagawa, H.; Nakayama, Y.; et al. Lactobacillus gasseri SBT2055 induces TGF- $\beta$ expression in dendritic cells and activates TLR2 signal to produce IgA in the small intestine. PLoS ONE 2014, 9, e105370. [CrossRef]

50. Gao, J.; Xu, K.; Liu, H.; Liu, G.; Bai, M.; Peng, C.; Li, T.; Yin, Y. Impact of the gut microbiota on intestinal immunity mediated by tryptophan metabolism. Front. Cell. Infect. Microbiol. 2018, 8, 8. [CrossRef] 